\title{
Tether-directed Selective Synthesis of Fulleropyrrolidine Bisadducts
}

\author{
Zhiguo Zhou,* David I. Schuster, Stephen R. Wilson
}

\section{Supporting Information}

NMR and mass data of bisadducts 6-15 and monoadducts 17-19..........S2

Figure $1{ }^{1} \mathrm{H}$ NMR $\left(\mathrm{CDCl}_{3}\right)$ spectrum of bisaldehyde $2 \ldots \ldots \ldots \ldots . . .56$

Figure $2{ }^{13} \mathrm{C}$ NMR $\left(\mathrm{CDCl}_{3}\right)$ spectrum of bisaldehyde $2 \ldots \ldots \ldots \ldots . . \mathrm{S} 6$

Figure $3{ }^{13} \mathrm{C}$ NMR $\left(\mathrm{CS}_{2}\right.$ /acetone) of fulleropyrrolidine bisadduct $5 \ldots \ldots \ldots . . . \mathrm{S} 7$

Figure $4{ }^{13} \mathrm{C}$ NMR $\left(\mathrm{CS}_{2} /\right.$ acetone $)$ of bisadduct 5, extended $\mathrm{sp}^{2}$ carbon area........S8

Figure $5{ }^{1} \mathrm{H}$ NMR (acetone/CS $\mathrm{CS}_{2}$ ) spectrum of bisadduct $6 \ldots \ldots \ldots \ldots \mathrm{S} 9$

Figure $6 \mathrm{COSY}$ (acetone/ $\mathrm{CS}_{2}$ ) spectrum of bisadduct $6 \ldots \ldots \ldots \ldots . . . \mathrm{S} 9$

Figure $7{ }^{13} \mathrm{C}$ NMR $\left(\mathrm{CS}_{2}\right.$ /acetone) of fulleropyrrolidine bisadduct $6 \ldots \ldots \ldots \ldots \ldots . . . . . .510$

Figure $8 \mathrm{COSY}$ (acetone/ $\mathrm{CS}_{2}$ ) spectrum of bisadduct $7 \ldots \ldots \ldots \ldots \ldots \mathrm{S} 11$

Figure $9{ }^{1} \mathrm{H}$ NMR $\left(\mathrm{CS}_{2}\right.$ /acetone) spectrum of bisadduct $10 \ldots \ldots \ldots \ldots . . . . .512$

Figure $10 \mathrm{COSY}$ NMR $\left(\mathrm{CS}_{2} /\right.$ acetone $)$ spectrum of bisadduct 10.............S12

Figure $11{ }^{1} \mathrm{H}$ NMR $\left(\mathrm{CS}_{2} /\right.$ acetone $)$ spectrum of bisadduct $15 \ldots \ldots \ldots \ldots . . . . . . . .313$

Figure 12 COSY NMR ( $\mathrm{CS}_{2} /$ acetone) spectrum of bisadduct $15 \ldots \ldots \ldots \ldots \ldots$. . . 13

Figure $13{ }^{1} \mathrm{H}$ NMR $\left(\mathrm{CS}_{2}\right.$ /acetone) of fulleropyrrolidine monoadduct 16.......... S14

Figure $14{ }^{13} \mathrm{C}$ NMR $\left(\mathrm{CS}_{2}\right.$ /acetone) of fulleropyrrolidine monoadduct 16..........S14

Figure 15 Maldi-tof mass spectrum of bisadduct 12...........S15

Computation method description and molecular modeling of bisadducts...........S16

Figure 16 Modeling of bisadducts derived from $p$-xylene tethered bisaldehydes...S16

Figure 17 Modeling of bisadducts derived from $o$-xylene tethered bisaldehydes....S17

Figure 18 Modeling of bisadducts derived from $m$-xylene tethered bisaldehydes...S17

Figure 19 Modeling of bisadducts derived from biphenyl tethered bisaldehydes...S18 
${ }^{1} \mathrm{H}$ NMR, ${ }^{13} \mathrm{C}$ NMR, 2D NMR, MALDI-MS data and/or selected spectra for compounds 1-19:

${ }^{1} \mathrm{H}$ NMR (400 MHz acetone- $\left.\mathrm{d}_{6} / \mathrm{CS}_{2} 1: 2\right)$ of bisadduct 6: $\delta 2.44(\mathrm{~s}, 6 \mathrm{H}), 4.12(\mathrm{~d}, 2 \mathrm{H})$, $4.53(\mathrm{~s}, 2 \mathrm{H}), 4.87(\mathrm{~d}, 2 \mathrm{H}), 5.11(\mathrm{~d}, 2 \mathrm{H}), 5.20(\mathrm{~d}, 2 \mathrm{H}), 6.79(\mathrm{~d}, 4 \mathrm{H}), 7.25(\mathrm{~s}, 4 \mathrm{H}), 7.30$ $(\mathrm{d}, 4 \mathrm{H}) ;{ }^{13} \mathrm{C}$ NMR $\left(100 \mathrm{MHz}\right.$ acetone- $\left.\mathrm{d}_{6} / \mathrm{CS}_{2} 1: 2\right)$ of bisadduct $6: \delta 40.2,67.5,69.1$, 70.4, 75.3, 84.5, 115.6, 127.4 (4C), 129.0, 137.4, 137.5 (2C), 137.6, 140.2, 141.2, $141.8,141.9,142.1,143.2,144.3,144.8,145.1,145.7,145.8,145.9,146.0$ (2C), 148.2, 148.8, 148.9, 149.0, 149.5, 149.6, 150.3, 153.8, 156.0, 157.9, 158.2 (2C); MALDI-MS: $\mathrm{m} / \mathrm{z} 1121.1\left(\mathrm{MH}^{+}\right)$.

${ }^{1} \mathrm{H}$ NMR (400 MHz acetone- $\left.\mathrm{d}_{6} / \mathrm{CS}_{2} 1: 2\right)$ of bisadduct 7: $\delta 2.38(\mathrm{~s}, 3 \mathrm{H}), 2.40(\mathrm{~s}, 3 \mathrm{H})$, $3.78(\mathrm{~d}, 1 \mathrm{H}), 3.80(\mathrm{~d}, 1 \mathrm{H}), 4.36(\mathrm{~s}, 1 \mathrm{H}), 4.39(\mathrm{~s}, 1 \mathrm{H}), 4.40(\mathrm{~d}, 1 \mathrm{H}), 4.48(\mathrm{~d}, 1 \mathrm{H}), 4.56$ $(\mathrm{d}, 1 \mathrm{H}), 5.10(\mathrm{~d}, 1 \mathrm{H}), 5.29(\mathrm{~d}, 1 \mathrm{H}), 5.40(\mathrm{~d}, 1 \mathrm{H}), 6.95(\mathrm{~d}, 2 \mathrm{H}), 7.09(\mathrm{~m}, 2 \mathrm{H}), 7.15(\mathrm{~m}$, 2H), $7.36(\mathrm{~d}, 2 \mathrm{H}), 7.42(\mathrm{~d}, 2 \mathrm{H}), 7.60(\mathrm{~d}, 2 \mathrm{H}) ;{ }^{13} \mathrm{C}$ NMR (100 MHz acetone- $\left.\mathrm{d}_{6} / \mathrm{CS}_{2} 1: 2\right)$ of bisadduct 7: $\delta 39.7,40.4,68.7,68.8,69.1,69.9$ (2C), 75.1, 75.8, 76.4, 83.1, 83.2, $115.3,120.8,126.1,129.0,129.6,129.7,129.8,130.8,131.6,132.1,135.6,135.9$, 136.6, 137.1, 138.6 (2C), 139.8 (2C), 140.1, 140.2, 140.9, 141.4, 141.7, 141.8, 142.0, $142.1,142.2$, 142.3, 142.8, 143.0, 143.1, 143.7, 143.9, 144.2, 144.4, 145.0, 145.1, 145.2 (2C), 145.3, 145.4, 145.7, 145.8 (2C), 145.9, 146.3, 146.5, 147.0, 147.1, 147.6, 147.7, 147.8, 148.0, 148.1 (2C), 148.2, 148.4, 148.7, 148.8, 149.3, 149.5, 150.4, 151.1, 151.7, 153.3, 153.4, 153.8 (2C), 154.6, 158.8 (2C), 159.0 (3C), 160.0, 160.8; MALDIMS: m/z $1120.7\left(\mathrm{M}^{+}\right)$.

${ }^{1} \mathrm{H}$ NMR (400 MHz acetone- $\mathrm{d}_{6} / \mathrm{CS}_{2}$ 1:2) of bisadduct 8: $\delta 2.44(\mathrm{~s}, 3 \mathrm{H}), 2.72(\mathrm{~s}, 3 \mathrm{H})$, $3.90(\mathrm{~d}, 1 \mathrm{H}), 4.01(\mathrm{~d}, 1 \mathrm{H}), 4.49(\mathrm{~s}, 1 \mathrm{H}), 4.50(\mathrm{~d}, 1 \mathrm{H}), 4.53(\mathrm{~s}, 1 \mathrm{H}), 4.69(\mathrm{~d}, 1 \mathrm{H}), 4.83$ $(\mathrm{d}, 1 \mathrm{H}), 5.18(\mathrm{~s}, 2 \mathrm{H}), 5.78(\mathrm{~d}, 1 \mathrm{H}), 6.75(\mathrm{~d}, 2 \mathrm{H}), 7.06(\mathrm{~d}, 2 \mathrm{H}), 7.10(\mathrm{~m}, 1 \mathrm{H}), 7.28(\mathrm{~m}$, 1H), $7.42(\mathrm{~m}, 2 \mathrm{H}), 7.54(\mathrm{~m}, 1 \mathrm{H}), 7.59(\mathrm{~m}, 2 \mathrm{H}), 7.81(\mathrm{~m}, 1 \mathrm{H}) ;{ }^{13} \mathrm{C} \mathrm{NMR}(100 \mathrm{MHz}$ acetone- $\left.\mathrm{d}_{6} / \mathrm{CS}_{2} 1: 2\right)$ of bisadduct $8: \delta 40.3,40.7,65.9,67.9,69.1,69.4,69.9,70.0,76.6$, $76.7,81.4,82.5,112.0,115.3,118.1,129.2,129.6,130.4,130.6,131.3,132.8$ (2C), 133.5 (2C), 134.1 (2C), 135.6, 136.4, 136.6, 137.7, 138.2 (2C), 139.3, 140.9, 141.4, 141.8, 142.1, 142.2, 142.6, 142.9 (2C), 143.1, 143.2, 143.3, 143.9, 144.2, 144.3, 144.8, 
$145.2,145.3,145.4(2 \mathrm{C}), 145.5,145.6,145.8,145.9,146.0,146.2,146.6,146.7,146.8$, 147.6, 147.8, 147.9, 148.1, 148.2, 148.3, 148.4, 148.6, 148.7 (2C), 148.9, 149.1, 149.6, 149.8, 150.6, 151.4, 152.4, 153.2, 154.2, 154.3, 154.8, 159.5 (2C), 159.7, 160.0 (2C), 161.1; MALDI-MS: m/z $1119.8\left(\mathrm{M}^{+}\right)$.

${ }^{1} \mathrm{H}$ NMR (400 MHz acetone- $\left.\mathrm{d}_{6} / \mathrm{CS}_{2} 1: 2\right)$ of bisadduct 9: $\delta 2.71(\mathrm{~s}, 3 \mathrm{H}), 4.15(\mathrm{~d}, 2 \mathrm{H})$, 4.59 (s, 2H), 4.72 (d, 2H), 4.80 (d, 2H), 4.84 (d, 2H), 6.59 (d, 2H), 6.61 (d, 2H), 7.00 (m, 2H), 7.09 (m, 2H), 7.19 (m, 2H), 7.38-7.43 (m, 2H); ${ }^{13} \mathrm{C}$ NMR (100 MHz acetone$\mathrm{d}_{6} / \mathrm{CS}_{2} 1: 2$ ) of bisadduct 9: $\delta 40.5,68.4,69.6,70.4,77.3,82.8,113.5,116.1,126.3$, $129.2,129.8,129.9,130.0$ (2C), 131.4, 132.2, 136.3, 137.1, 137.9, 140.2, 142.1, 142.2, $142.3,142.4,143.1,144.3,144.7,145.0,145.6,145.9,146.0,146.1,148.3,149.0$, 149.2, 149.4, 149.9, 150.0, 150.8, 154.9, 155.8, 158.3, 158.5, 159.8 (2C); MALDI-MS: $\mathrm{m} / \mathrm{z} 1120.4\left(\mathrm{M}^{+}\right)$.

${ }^{1} \mathrm{H}$ NMR (400 MHz acetone- $\left.\mathrm{d}_{6} / \mathrm{CS}_{2} 1: 2\right)$ of bisadduct 10: $\delta 2.73(\mathrm{~s}, 3 \mathrm{H}), 2.79(\mathrm{~s}, 3 \mathrm{H})$, $3.91(\mathrm{~d}, 1 \mathrm{H}), 4.29(\mathrm{~d}, 1 \mathrm{H}), 4.30(\mathrm{~s}, 1 \mathrm{H}), 4.50(\mathrm{~d}, 1 \mathrm{H}), 4.62(\mathrm{~d}, 1 \mathrm{H}), 4.75(\mathrm{~s}, 1 \mathrm{H}), 4.88$ $(\mathrm{d}, 1 \mathrm{H}), 4.90(\mathrm{~d}, 1 \mathrm{H}), 5.04(\mathrm{~d}, 1 \mathrm{H}), 5.10(\mathrm{~d}, 1 \mathrm{H}), 6.62(\mathrm{~d}, 1 \mathrm{H}), 6.81(\mathrm{~d}, 1 \mathrm{H}), 7.01(\mathrm{~m}$, 1H), $7.09(\mathrm{~m}, 1 \mathrm{H}), 7.21-7.30(\mathrm{~m}, 5 \mathrm{H}), 7.40(\mathrm{~d}, 1 \mathrm{H}), 7.49(\mathrm{~d}, 1 \mathrm{H}), 7.629 \mathrm{~d}, 1 \mathrm{H}) ;{ }^{13} \mathrm{C}$ NMR (100 MHz acetone- $\left.\mathrm{d}_{6} / \mathrm{CS}_{2} 1: 2\right)$ of bisadduct 10: $\delta$ 40.2, 40.7, 68.3, 69.0, 69.2, 70.8, 71.3, 71.9, 77.1, 78.2, 81.1, 84.8, 115.4, 116.8, 126.4, 128.3 (2C), 128.7 (2C), $129.3,130.0$ (2C), 130.1, 130.7, 130.8, 130.9, 131.8, 135.0, 135.1, 133.9, 137.8, 138.3, 138.5 (2C), 139.2, 140.1, 141.4, 141.6, 142.0, 142.1, 142.3, 142.5, 142.9, 143.0, 143.7, 144.3, 144.5, 144.6, 144.7, 144.8, 144.9, 145.4, 145.5, 145.7, 145.8, 145.9, 146.0 (3C), 146.1 (3C), 146.3, 147.2, 148.7, 148.8, 148.9, 149.0, 149.2, 149.4, 149.6, 149.7, 149.9, $150.1,150.2,150.3,154.4,154.9,155.4,155.8,157.7,157.8,158.5,158.7,160.1(2 \mathrm{C})$, 162.1 (2C); MALDI-MS: m/z $1120.3\left(\mathrm{M}^{+}\right)$.

${ }^{1} \mathrm{H}$ NMR (400 MHz acetone- $\left.\mathrm{d}_{6} / \mathrm{CS}_{2} 1: 2\right)$ of bisadduct 11: $\delta 2.52(\mathrm{~s}, 6 \mathrm{H}), 4.10(\mathrm{~d}, 2 \mathrm{H})$, $4.50(\mathrm{~s}, 2 \mathrm{H}), 4.79$ (d, 2H), 4.99 (s, 4H), 6.56 (d, 4H), 7.02 (m, 2H), 7.11 (m, 2H), 7.19$7.23(\mathrm{~m}, 3 \mathrm{H}), 7.32(\mathrm{~s}, 1 \mathrm{H})$; MALDI-MS: m/z $1121.2\left(\mathrm{MH}^{+}\right) .{ }^{1} \mathrm{H}$ NMR (400 MHz acetone- $\left.\mathrm{d}_{6} / \mathrm{CS}_{2} 1: 2\right)$ of bisadduct 12: $\delta 2.35(\mathrm{~s}, 3 \mathrm{H}), 2.41(\mathrm{~s}, 3 \mathrm{H}), 3.78(\mathrm{~d}, 1 \mathrm{H}), 3.81(\mathrm{~d}$, 1H), $4.30(\mathrm{~s}, 1 \mathrm{H}), 4.34(\mathrm{~s}, 1 \mathrm{H}), 4.42(\mathrm{~d}, 1 \mathrm{H}), 4.51(\mathrm{~d}, 1 \mathrm{H}), 4.75(\mathrm{~d}, 1 \mathrm{H}), 4.92(\mathrm{~d}, 1 \mathrm{H})$, 
$5.14(\mathrm{~d}, 1 \mathrm{H}), 5.35(\mathrm{~d}, 1 \mathrm{H}), 6.62(\mathrm{~d}, 2 \mathrm{H}), 6.82(\mathrm{~m}, 2 \mathrm{H}), 6.94(\mathrm{~m}, 2 \mathrm{H}), 7.20(\mathrm{~s}, 1 \mathrm{H})$, 7.36-7.42 (m, 3H), 7.80 (s, 2H); MALDI-MS: m/z $1121.1\left(\mathrm{MH}^{+}\right) .{ }^{1} \mathrm{H}$ NMR (400 MHz acetone- $\left.\mathrm{d}_{6} / \mathrm{CS}_{2} 1: 2\right)$ of bisadduct 13: $\delta 2.35(\mathrm{~s}, 3 \mathrm{H}), 2.41(\mathrm{~s}, 3 \mathrm{H}), 3.89(\mathrm{~d}, 1 \mathrm{H}), 4.24(\mathrm{~d}$, 1H), $4.29(\mathrm{~s}, 1 \mathrm{H}), 4.65(\mathrm{~s}, 1 \mathrm{H}), 4.70(\mathrm{~d}, 1 \mathrm{H}), 4.86(\mathrm{~d}, 1 \mathrm{H}), 4.90(\mathrm{~d}, 1 \mathrm{H}), 4.98(\mathrm{~d}, 1 \mathrm{H})$, $5.00(\mathrm{~s}, 1 \mathrm{H}), 5.06(\mathrm{~d}, 1 \mathrm{H}), 6.59(\mathrm{~m}, 2 \mathrm{H}), 6.80(\mathrm{~m}, 2 \mathrm{H}), 6.91(\mathrm{~s}, 1 \mathrm{H}), 7.14(\mathrm{~m}, 2 \mathrm{H}), 7.28$ -7.35 (m, 3H), $7.84(\mathrm{~m}, 2 \mathrm{H})$; MALDI-MS: m/z $1120.8\left(\mathrm{M}^{+}\right)$.

${ }^{1} \mathrm{H}$ NMR (400 MHz, CS 2 /acetone- $\mathrm{d}_{6}$ ) of 14: $\delta 2.83(\mathrm{~s}, 3 \mathrm{H}), 2.92(\mathrm{~s}, 3 \mathrm{H}), 4.06(\mathrm{~d}, 1 \mathrm{H})$, $4.30(\mathrm{~d}, 1 \mathrm{H}), 4.70(\mathrm{~s}, 1 \mathrm{H}), 4.82(\mathrm{~d}, 1 \mathrm{H}), 5.09(\mathrm{~s}, 1 \mathrm{H}), 5.15(\mathrm{~d}, 1 \mathrm{H}), 5.20(\mathrm{~d}, 1 \mathrm{H}), 5.26$ $(\mathrm{m}, 3 \mathrm{H}), 6.52(\mathrm{~d}, 1 \mathrm{H}), 6.67(\mathrm{~d}, 1 \mathrm{H}), 6.97-7.06(\mathrm{~m}, 6 \mathrm{H}), 7.12(\mathrm{~d}, 1 \mathrm{H}), 7.18-7.24(\mathrm{~m}$, $4 \mathrm{H}), 7.48(\mathrm{~d}, 1 \mathrm{H}), 7.64(\mathrm{~d}, 1 \mathrm{H}), 8.05(\mathrm{~d}, 1 \mathrm{H}) ;{ }^{13} \mathrm{C}$ NMR $\left(100 \mathrm{MHz}, \mathrm{CS}_{2} /\right.$ acetone- $\left._{6}\right)$ of 14: $\delta 40.2,40.4,67.7,67.9,68.2,68.3,70.5$ (2C), 76.7, 77.2, 82.7, 82.9, 126.1, 126.8, $16.9,127.3,128.2$, 128.9, 129.6, 129.7, 130.2, 130.3, 130.6, 130.7, 131.2, 133.8, 134.5, 134.8, 134.9, 136.8 (2C), 136.9, 138.9 (2C), 139.1, 139.2 (2C), 140.2, 140.3, 140.8, $141.3,141.8,141.9$ (2C), 142.0, 142.1, 142.3, 142.8, 142.9, 143.0, 143.2, 143.4, 143.6, $144.0,144.1,144.3,144.5,144.6,144.7,145.1,145.3,145.7,145.8,145.9,146.0$, 146.1, 146.2, 146.3, 146.5, 146.6, 146.7, 146.9 (2C), 147.0, 147.2, 147.5, 147.6, 147.8, 148.5, 148.7, 149.0, 150.1, 151.9, 152.4, 152.8, 152.9, 154.2, 155.1, 157.8 (2C), 158.2 (2C), 158.4, 158.5, MALDI-MS: m/z $1120.1\left(\mathrm{M}^{+}\right)$. ${ }^{1} \mathrm{H}$ NMR (400 MHz, $\mathrm{CS}_{2} /$ acetone$\left.\mathrm{d}_{6}\right)$ of 15: $\delta 2.81(\mathrm{~s}, 3 \mathrm{H}), 2.96(\mathrm{~s}, 3 \mathrm{H}), 4.30(\mathrm{~d}, 1 \mathrm{H}), 4.40(\mathrm{~d}, 1 \mathrm{H}), 4.79(\mathrm{~d}, 1 \mathrm{H}), 4.83(\mathrm{~s}$, 1H), $4.90(\mathrm{~d}, 1 \mathrm{H}), 5.00(\mathrm{~s}, 1 \mathrm{H}), 5.10-5.18(\mathrm{~m}, 3 \mathrm{H}), 5.32(\mathrm{~d}, 1 \mathrm{H}), 6.25(\mathrm{~d}, 1 \mathrm{H}), 6.70(\mathrm{~d}$, 1H), 6.80-6.91 (m, 4H), 6.94-7.03 (m, 4H), 7.35 (d, 1H), $7.40(\mathrm{~d}, 1 \mathrm{H}), 7.52(\mathrm{~d}, 1 \mathrm{H})$, $7.59(\mathrm{~d}, 1 \mathrm{H}), 7.64(\mathrm{~d}, 1 \mathrm{H}), 7.84(\mathrm{~d}, 1 \mathrm{H}) ;$ MALDI-MS: m/z $1120.1\left(\mathrm{M}^{+}\right)$.

${ }^{1} \mathrm{H}$ NMR (400 MHz $\mathrm{CDCl}_{3}$ ) of 17: $\delta 2.76(\mathrm{~s}, 3 \mathrm{H}), 4.22(\mathrm{~d}, 1 \mathrm{H}), 4.84(\mathrm{~s}, 1 \mathrm{H}), 4.92(\mathrm{~d}$, 1H), $5.11(\mathrm{~s}, 2 \mathrm{H}), 5.23(\mathrm{~s}, 2 \mathrm{H}), 6.93(\mathrm{~d}, 2 \mathrm{H}), 6.99(\mathrm{~d}, 2 \mathrm{H}), 7.32(\mathrm{~m}, 2 \mathrm{H}), 7.43(\mathrm{~m}, 2 \mathrm{H})$, $7.65(\mathrm{~m}, 2 \mathrm{H}), 7.75(\mathrm{~d}, 2 \mathrm{H}), 9.81(\mathrm{~s}, 1 \mathrm{H}) ;{ }^{13} \mathrm{C} \mathrm{NMR}\left(100 \mathrm{MHz}^{\mathrm{CDCl}} \mathrm{CD}_{3}\right)$ of 17: $\delta 40.2$, 68.2, 68.3, 68.9, 70.0, 83.1 (2C), 116.0, 128.7 (2C), 128.9, 129.4, 129.6, 130.3, 130.6, 132.0 (2C), 134.4, 134.8, 135.8, 135.9, 136.6, 136.9, 139.6, 140.0, 140.2, 140.3, 141.6, 141.8, 141.9, 142.0 (2C), 142.1 (2C), 142.2 (2C), 142.3, 142.6 (2C), 142.7, 142.8, $143.1,143.2,144.4,144.5,144.7,144.8,145.2$ (2C), 145.3 (2C), 145.4, 145.5 (2C), 145.6 (2C), 145.7, 145.8, 146.0 (2C), 146.1, 146.2, 146.3 (2C), 146.4 (3C), 146.5, 
146.8, 147.3 (2C), 153.2, 153.4, 154.1, 156.2, 158.6, 163.4, 189.8; MALDI-MS: m/z $1066.1\left(\mathrm{M}^{+}\right)$.

${ }^{1} \mathrm{H}$ NMR (400 MHz $\mathrm{CDCl}_{3}$ ) of 18: $\delta 2.80$ (s, 3H), 4.25 (d, 1H), 4.90 (s, 1H), 5.00 (d, $1 \mathrm{H}), 5.05(\mathrm{~s}, 2 \mathrm{H}), 5.18(\mathrm{~s}, 2 \mathrm{H}), 6.95(\mathrm{~d}, 2 \mathrm{H}), 7.05(\mathrm{~d}, 2 \mathrm{H}), 7.10(\mathrm{~m}, 1 \mathrm{H}), 7.34(\mathrm{~m}, 3 \mathrm{H})$, $7.49(\mathrm{~s}, 1 \mathrm{H}), 7.66(\mathrm{~m}, 1 \mathrm{H}), 7.72(\mathrm{~d}, 2 \mathrm{H}), 9.79(\mathrm{~s}, 1 \mathrm{H}) ;{ }^{13} \mathrm{C} \mathrm{NMR}\left(100 \mathrm{MHz} \mathrm{CDCl}_{3}\right)$ of 18: $\delta 39.9,69.1,69.7,69.9,70.1,77.6,83.2,115.5,125.5,126.8,127.1,127.3,128.4$, 128.9, 129.1, 129.3, 130.6, 131.8 (2C), 136.0, 136.1, 136.7, 136.8 (2C), 137.0, 137.7 (2C), 139.6, 140.1, 140.2, 140.3, 141.7, 141.8, 141.9, 142.1, 142.2, 142.3 (2C), 142.4 (2C), 142.5 (2C), 142.7 (3C), 142.8, 143.1, 143.3, 144.5, 144.6, 144.8, 144.9, 145.2, 145.3 (2C), 145.4 (2C), 145.5, 145.6, 145.7 (2C), 145.8, 145.9, 146.1 (2C), 146.2 (2C), 146.3 (2C), 146.4 (2C), 146.6, 146.8, 147.0, 147.4 (2C), 153.9, 154.3, 156.7, 159.0, 163.7, 189.2; MALDI-MS: m/z $1066.2\left(\mathrm{M}^{+}\right)$.

${ }^{1} \mathrm{H}$ NMR (400 MHz $\left.\mathrm{CDCl}_{3}\right)$ of 19: $\delta 2.73(\mathrm{~s}, 3 \mathrm{H}), 4.23(\mathrm{~d}, 1 \mathrm{H}), 4.85(\mathrm{~s}, 1 \mathrm{H}), 4.93(\mathrm{~d}$, 1H), $5.02(\mathrm{~s}, 2 \mathrm{H}), 5.16(\mathrm{~s}, 2 \mathrm{H}), 6.92(\mathrm{~d}, 2 \mathrm{H}), 7.00(\mathrm{~d}, 2 \mathrm{H}), 7.08(\mathrm{~m}, 1 \mathrm{H}), 7.40(\mathrm{dd}, 4 \mathrm{H})$, $7.50(\mathrm{dd}, 4 \mathrm{H}), 7.51(\mathrm{~m}, 1 \mathrm{H}), 7.52(\mathrm{~d}, 2 \mathrm{H}), 9.87(\mathrm{~s}, 1 \mathrm{H}) ;{ }^{13} \mathrm{C} \mathrm{NMR}\left(100 \mathrm{MHz} \mathrm{CDCl}_{3}\right)$ of 19: $\delta 40.3,69.8,70.5,70.8$ (2C), 78.4, 83.9, 116.8, 127.5, 128.1 (2C), $128.2(2 \mathrm{C})$, 129.0 (2C), 129.1 (2C), 129.2, 129.9, 130.1, 131.4 (2C), 132.6 (2C), 136.5, 136.8, $136.9,137.3,137.6,137.9,140.5,140.9,141.1$ (2C), 141.2, 141.4, 142.5, 142.7, 142.8, 142.9 (2C), 143.0, 143.1 (2C), 143.2 (2C), 143.3 (2C), 144.0, 144.1, 145.4, 145.6, $145.7,146.1,146.2$ (3C), 146.3 (2C), 146.5, 146.6 (2C), 146.7, 146.8, 146.9 (2C), 147.0 (2C), 147.1, 147.2 (2C), 147.3, 147.5, 147.6, 147.9, 148.2, 154.8, 155.3, 157.6, 160.0, 164.6, 190.0; MALDI-MS: m/z $1145.1\left(\mathrm{M}^{+}\right)$. 


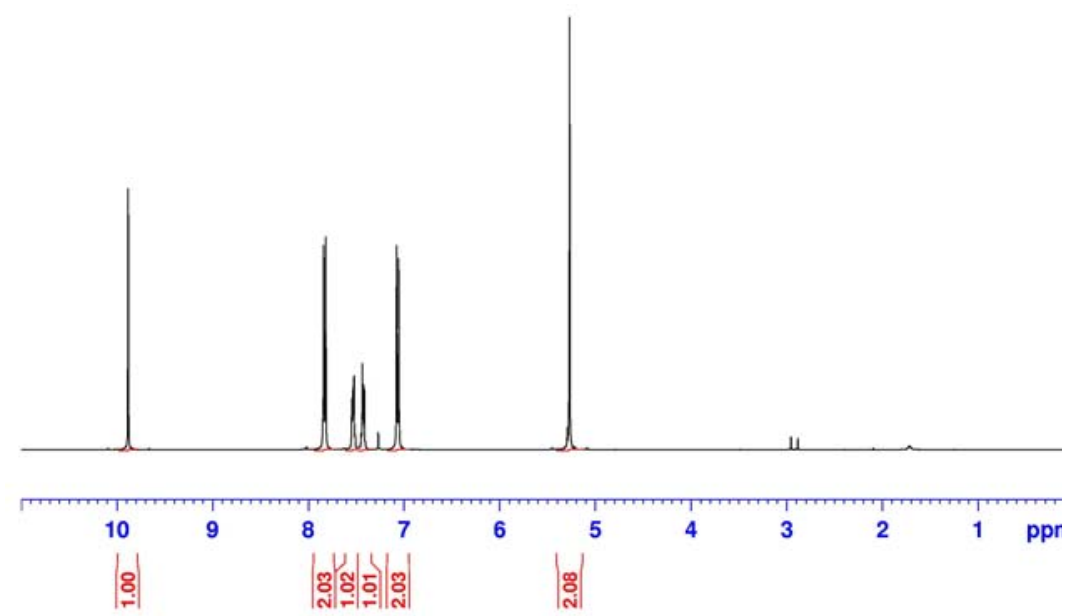

Figure $1{ }^{1} \mathrm{H}$ NMR $\left(\mathrm{CDCl}_{3}\right)$ spectrum of bisaldehyde 2

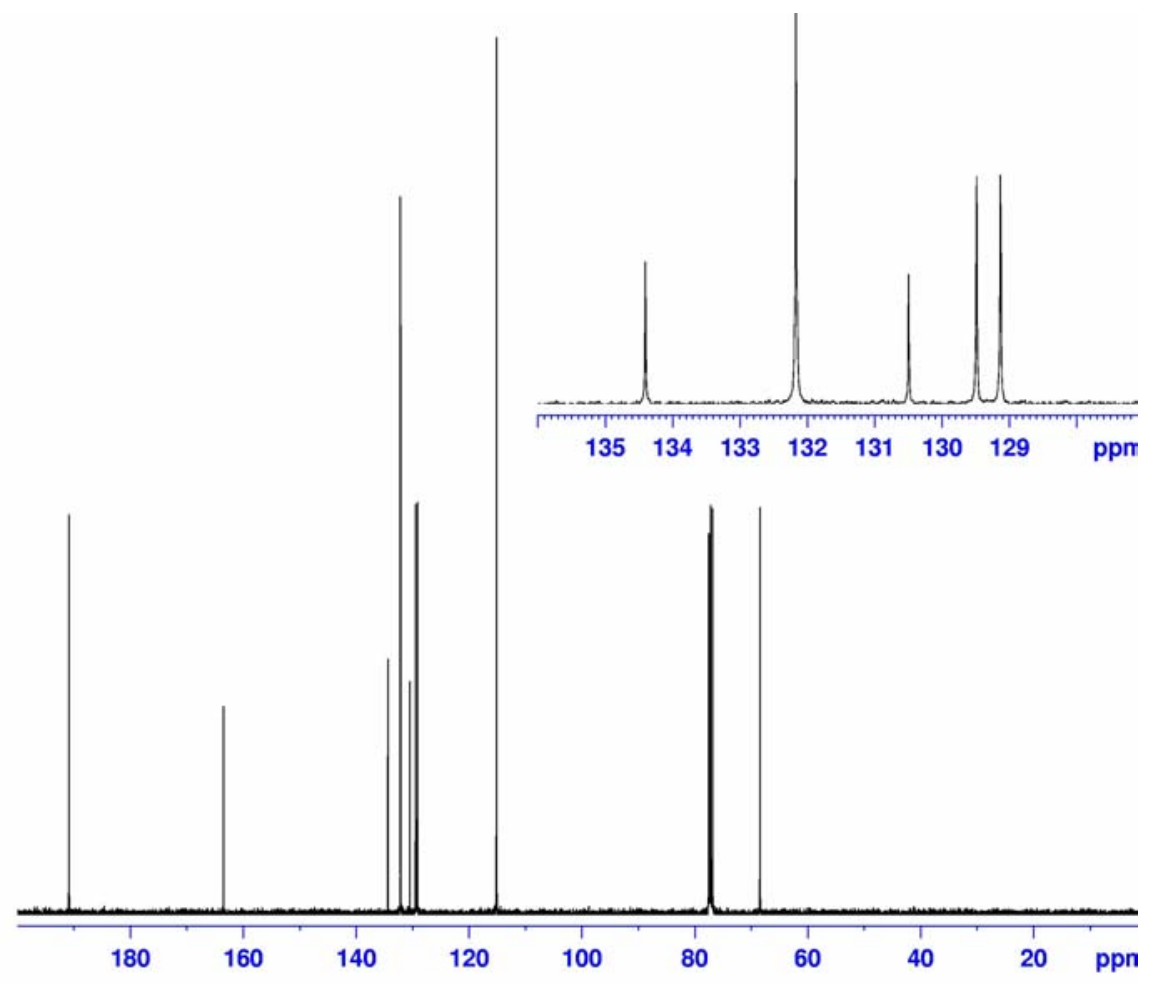

Figure $2{ }^{13} \mathrm{C}$ NMR $\left(\mathrm{CDCl}_{3}\right)$ spectrum of bisaldehyde $\mathbf{2}$ 

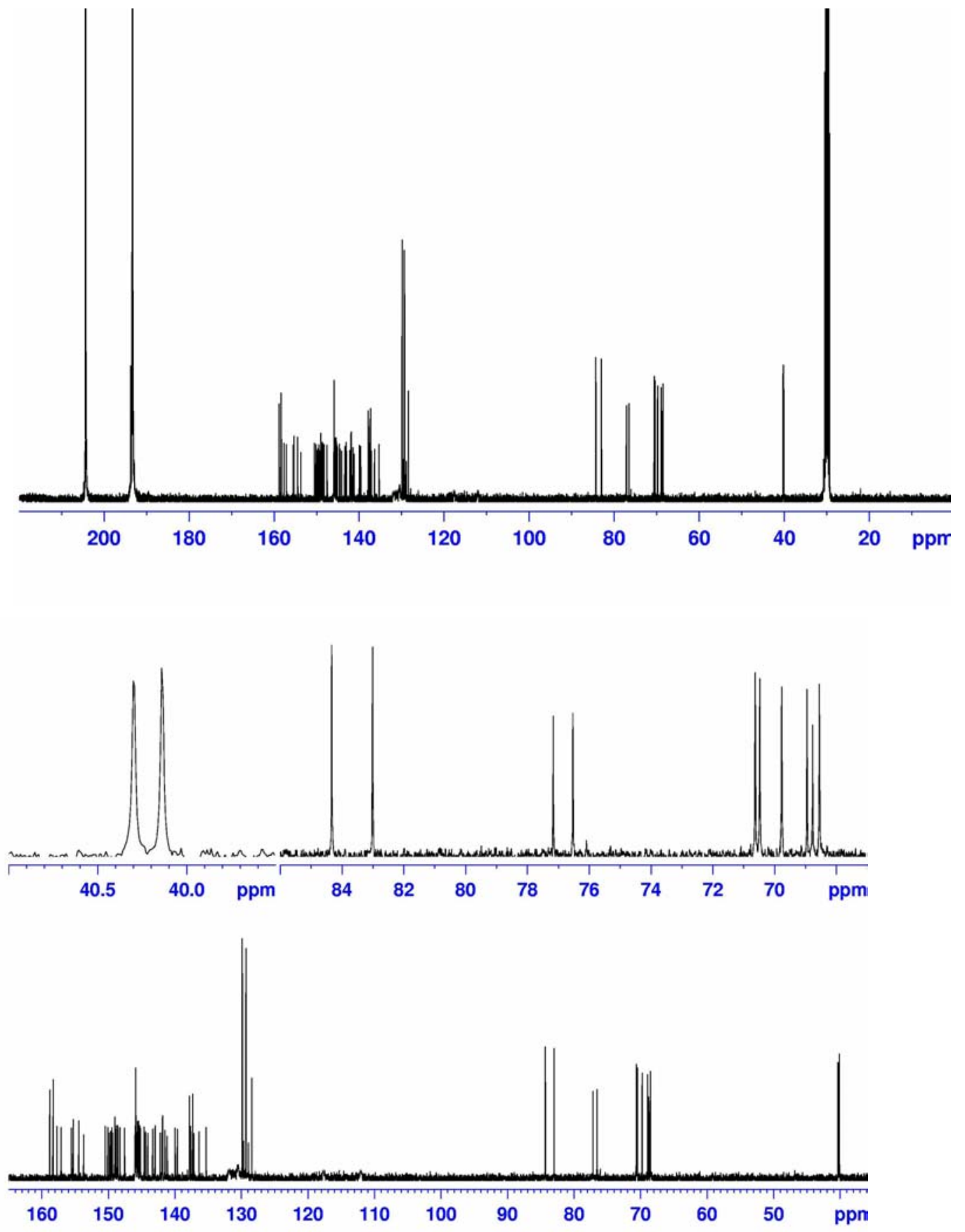

Figure $3{ }^{13} \mathrm{C}$ NMR $\left(\mathrm{CS}_{2}\right.$ /acetone) of fulleropyrrolidine bisadduct 5 (see extended $s p^{2}$ carbon area on next page) 

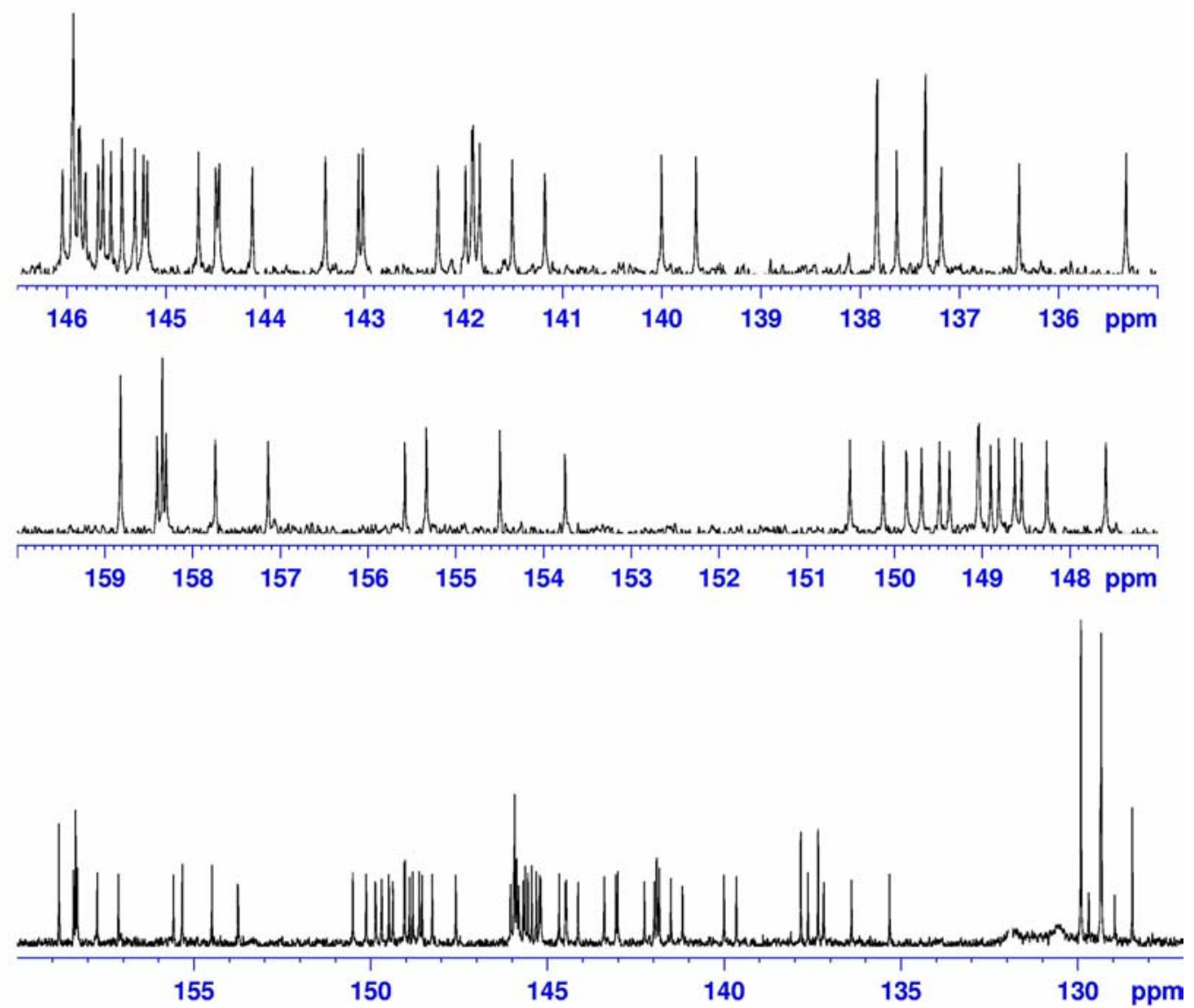

Figure $4{ }^{13} \mathrm{C}$ NMR $\left(\mathrm{CS}_{2}\right.$ /acetone) of fulleropyrrolidine bisadduct 5 , extended $s p^{2}$ carbon area 


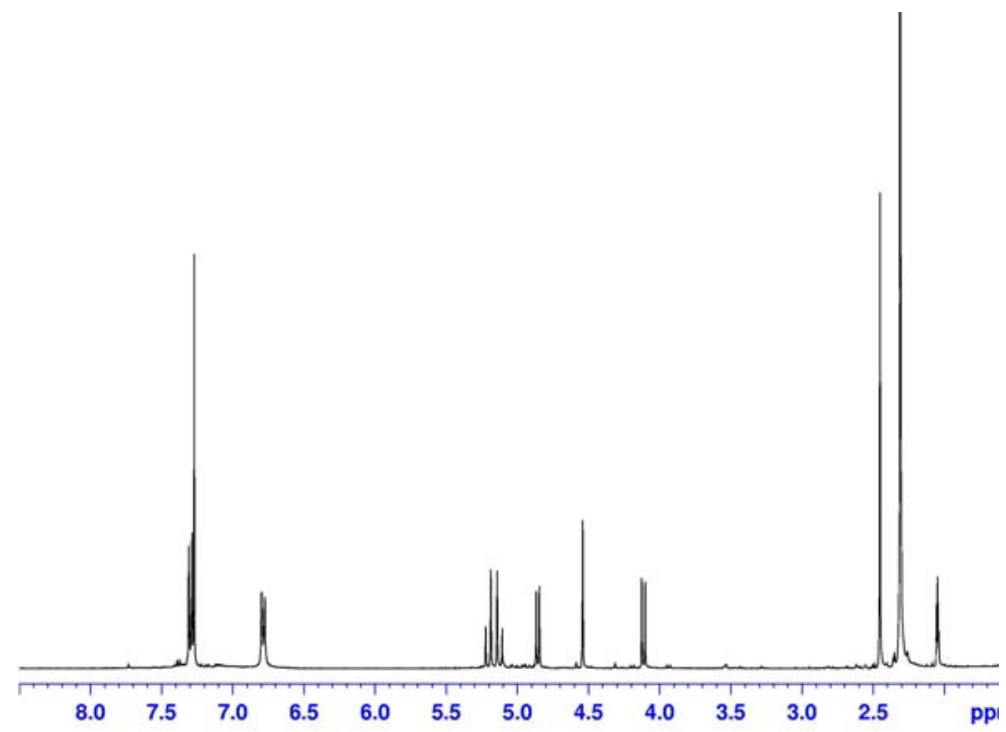

Figure $5{ }^{1} \mathrm{H}$ NMR (acetone/CS 2 ) spectrum of bisadduct 6

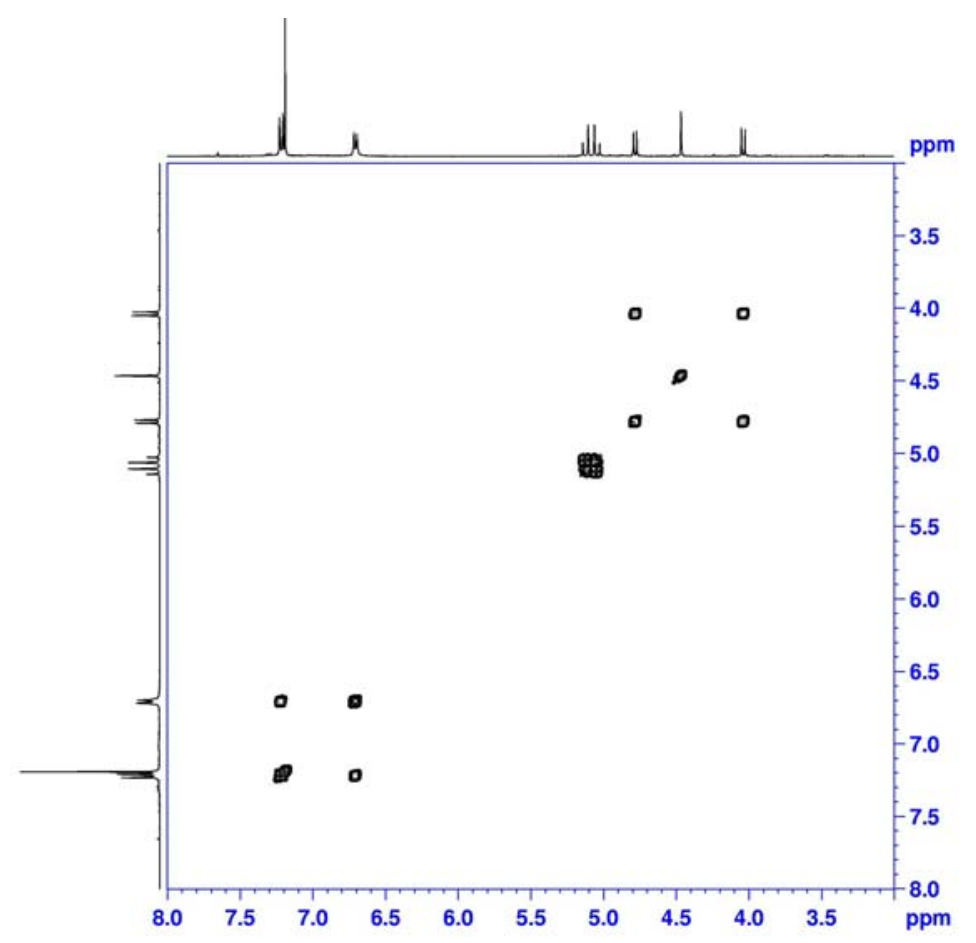

Figure 6 COSY (acetone/ $\mathrm{CS}_{2}$ ) spectrum of bisadduct 6 

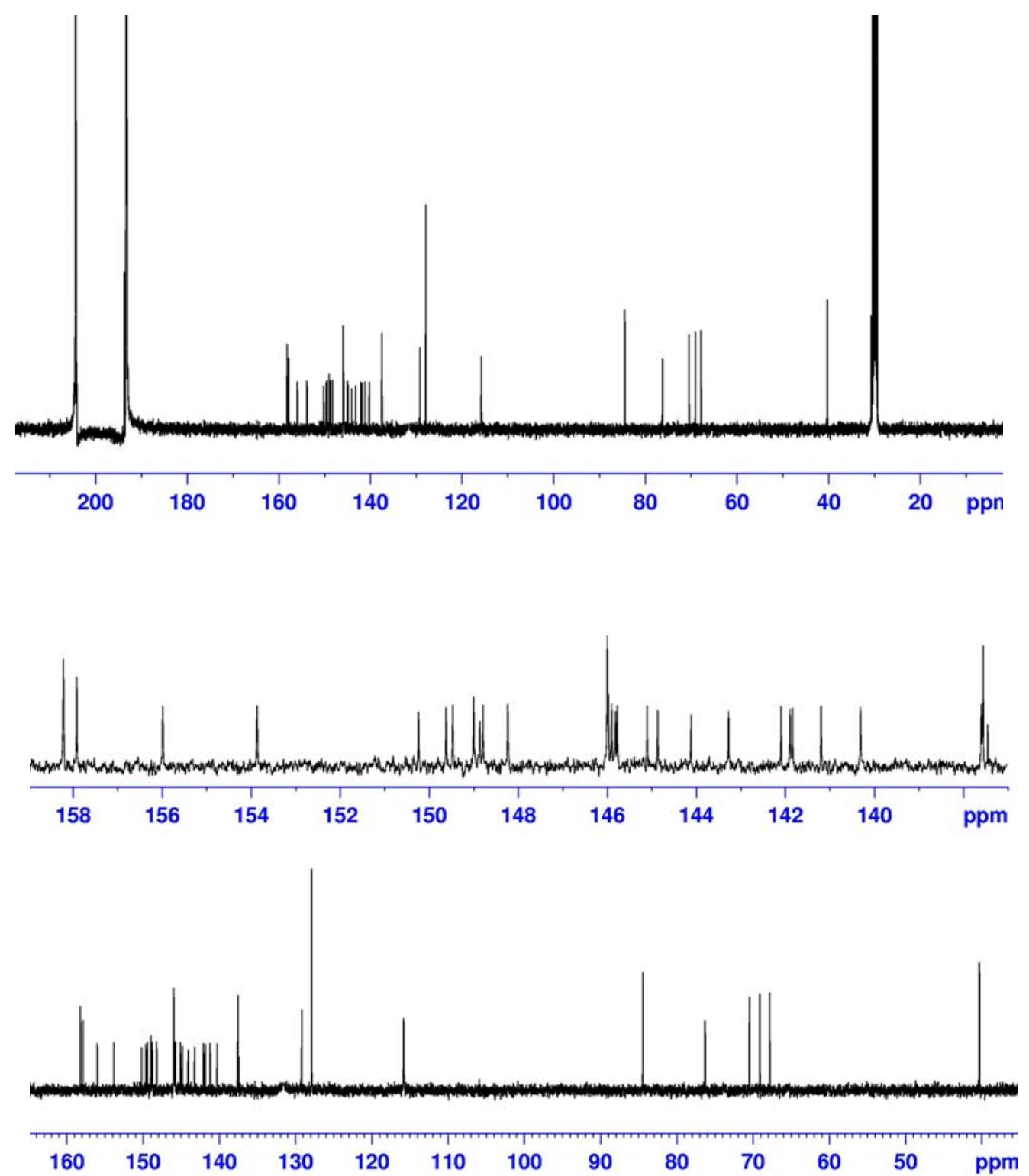

Figure $7{ }^{13} \mathrm{C}$ NMR $\left(\mathrm{CS}_{2} /\right.$ acetone $)$ of fulleropyrrolidine bisadduct 6 


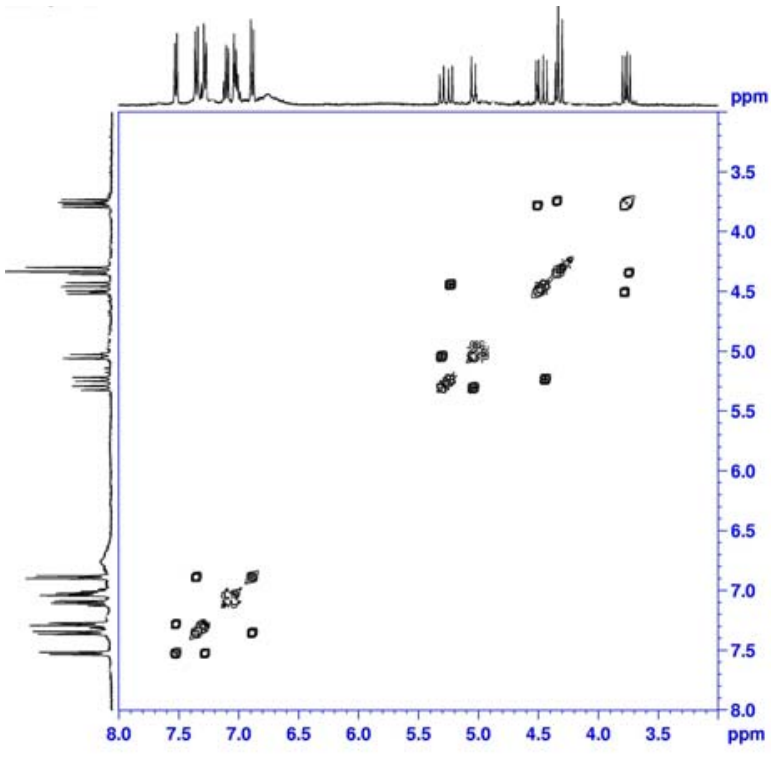

Figure $8 \mathrm{COSY}$ (acetone/CS 2 ) spectrum of bisadduct 7 


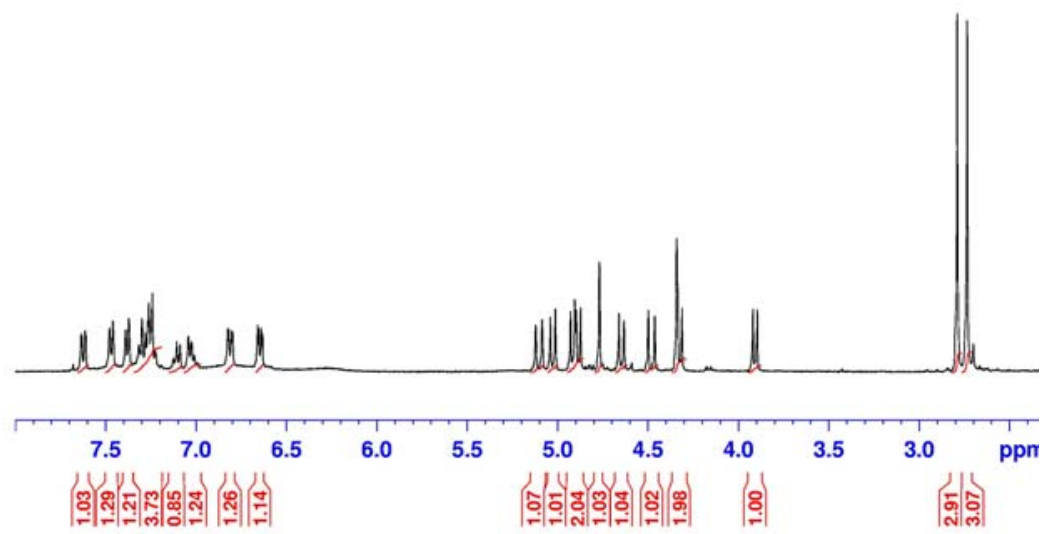

Figure $9{ }^{1} \mathrm{H}$ NMR $\left(\mathrm{CS}_{2} /\right.$ acetone $)$ spectrum of bisadduct 10

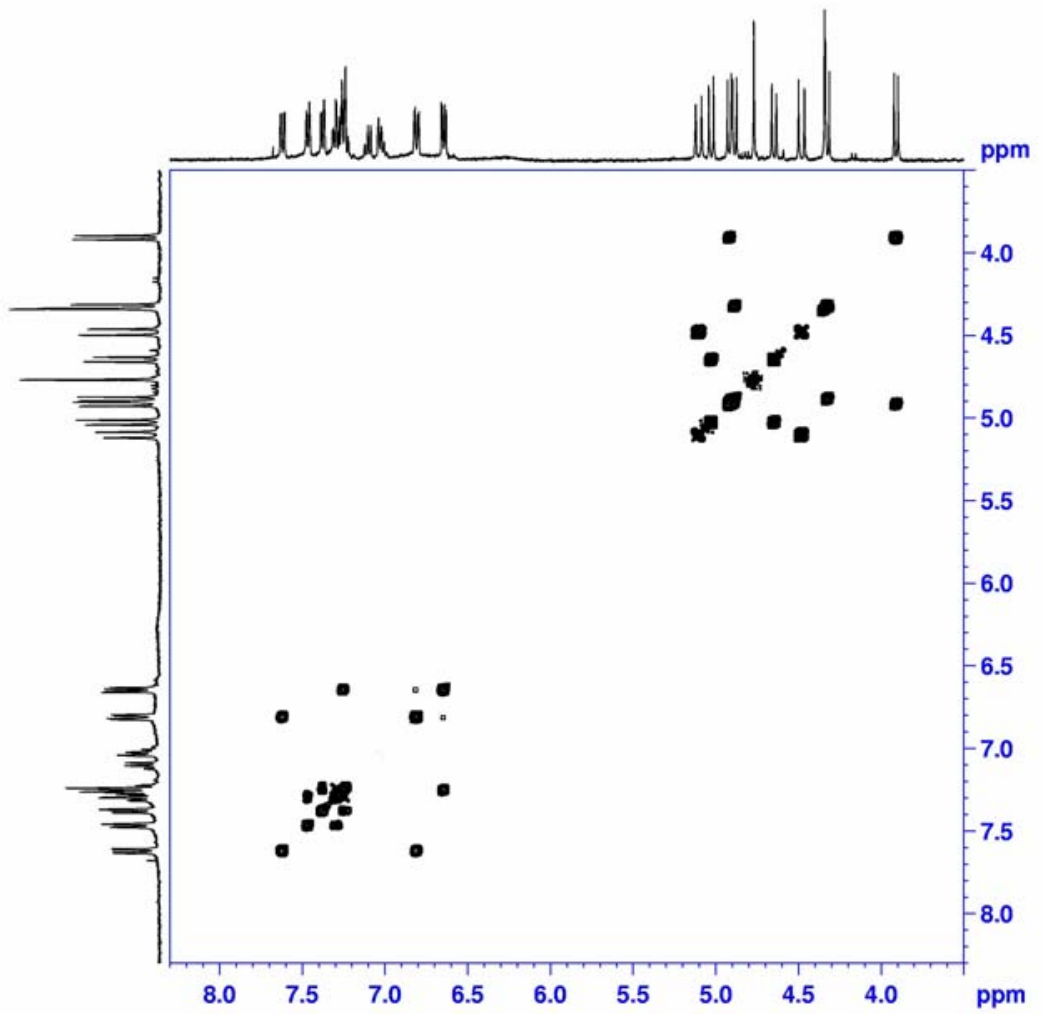

Figure 10 COSY NMR $\left(\mathrm{CS}_{2} /\right.$ acetone $)$ spectrum of bisadduct 10 


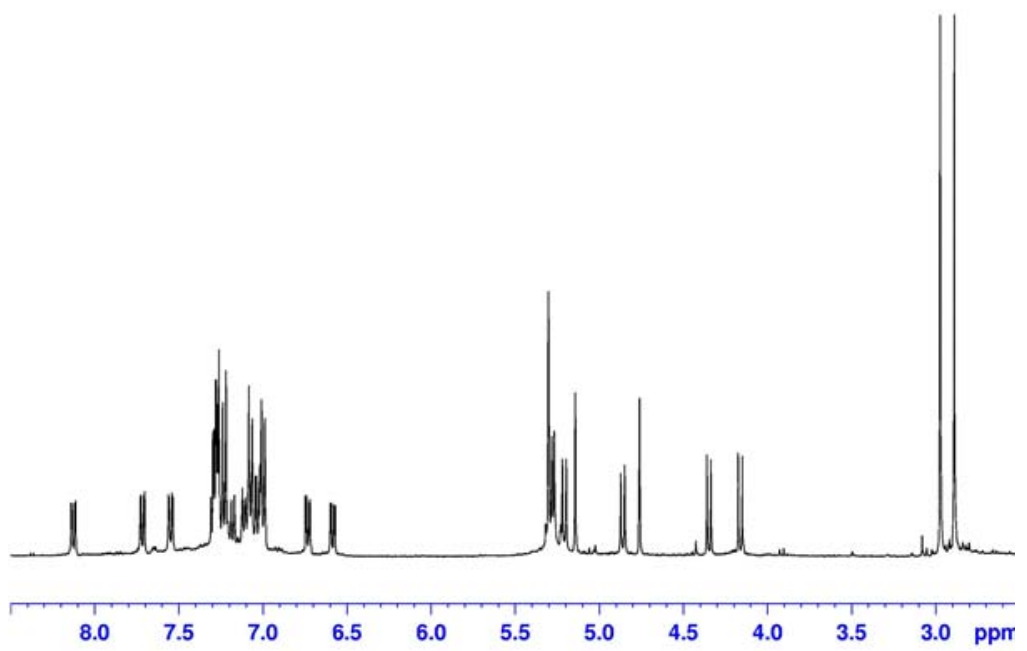

Figure $11{ }^{1} \mathrm{H}$ spectrum $\left(\mathrm{CS}_{2} /\right.$ acetone $)$ of bisadduct 15

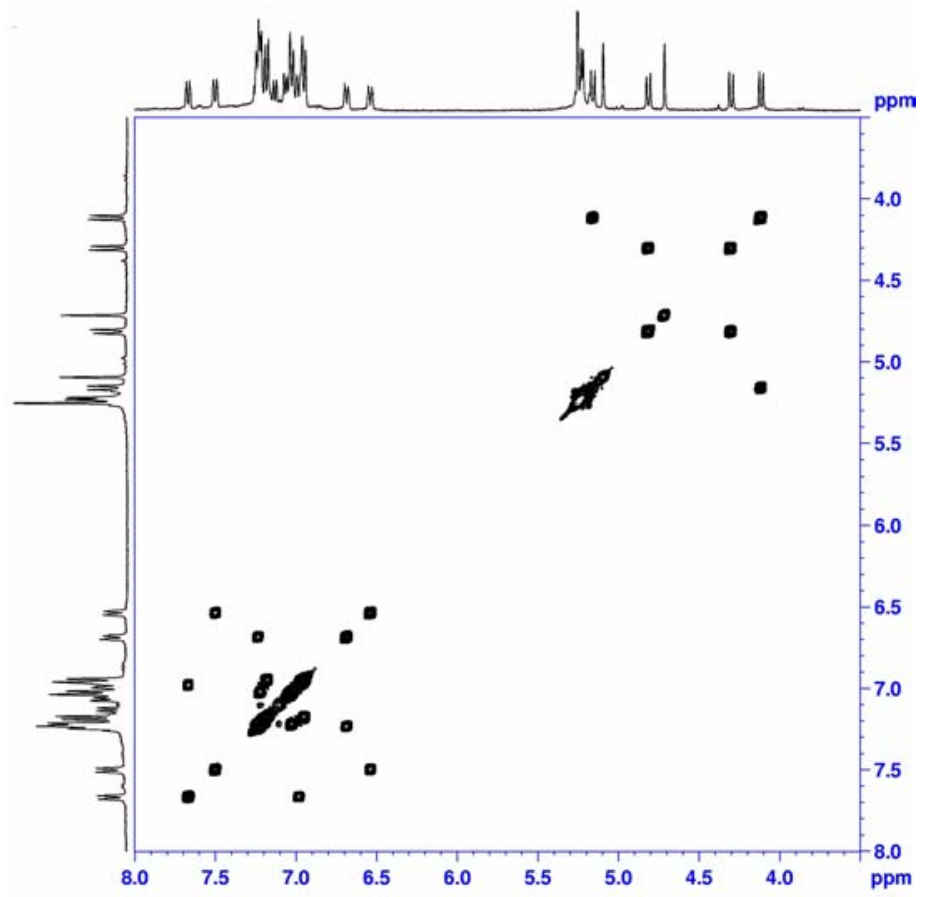

Figure $12 \mathrm{COSY}$ spectrum $\left(\mathrm{CS}_{2} /\right.$ acetone $)$ of bisadduct 15 


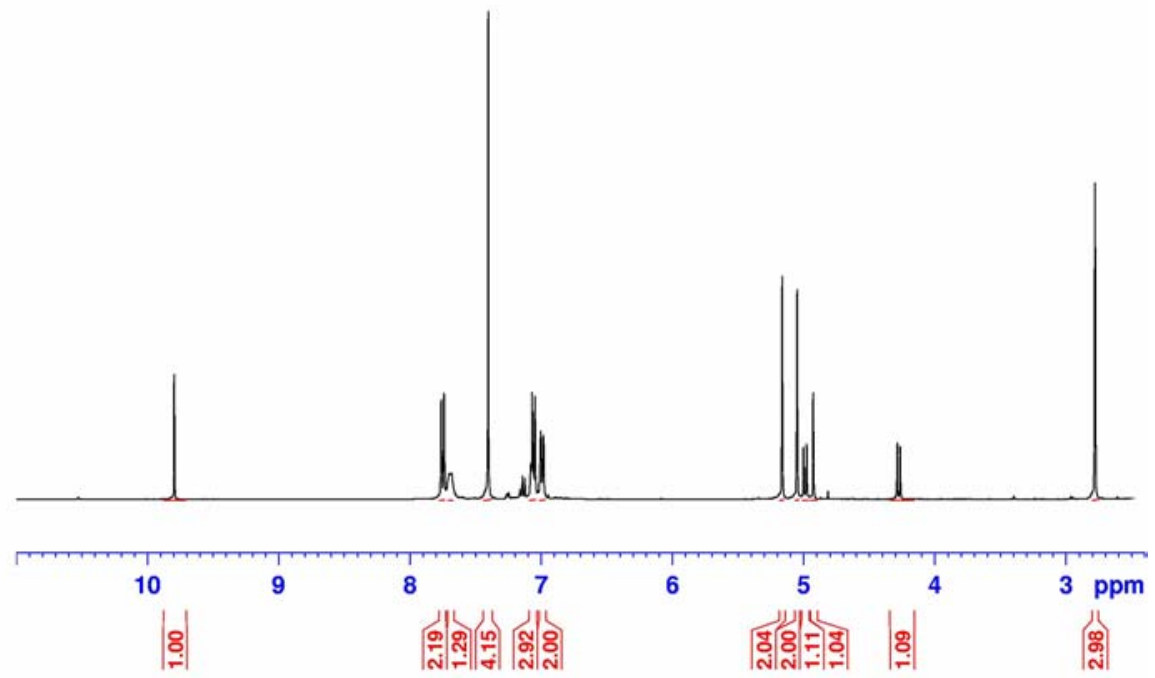

Figure $13{ }^{1} \mathrm{H}\left(\mathrm{CS}_{2} /\right.$ acetone $)$ of fulleropyrrolidine monoadduct 16

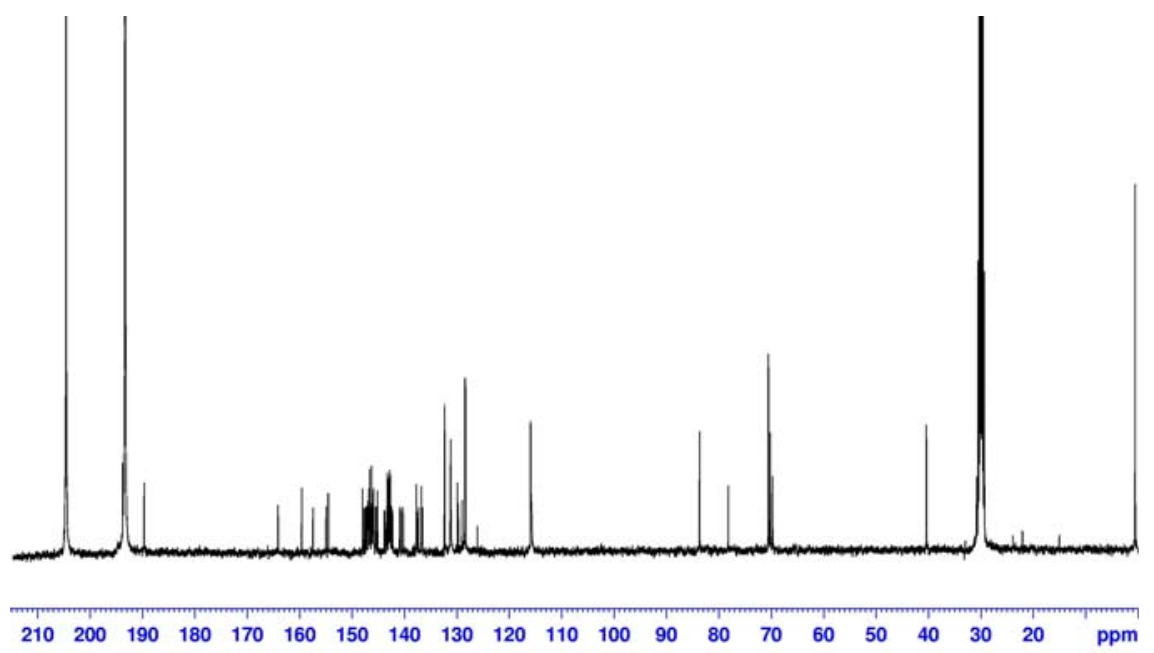

Figure $14{ }^{13} \mathrm{C}$ NMR $\left(\mathrm{CS}_{2} /\right.$ acetone $)$ of fulleropyrrolidine monoadduct 16 


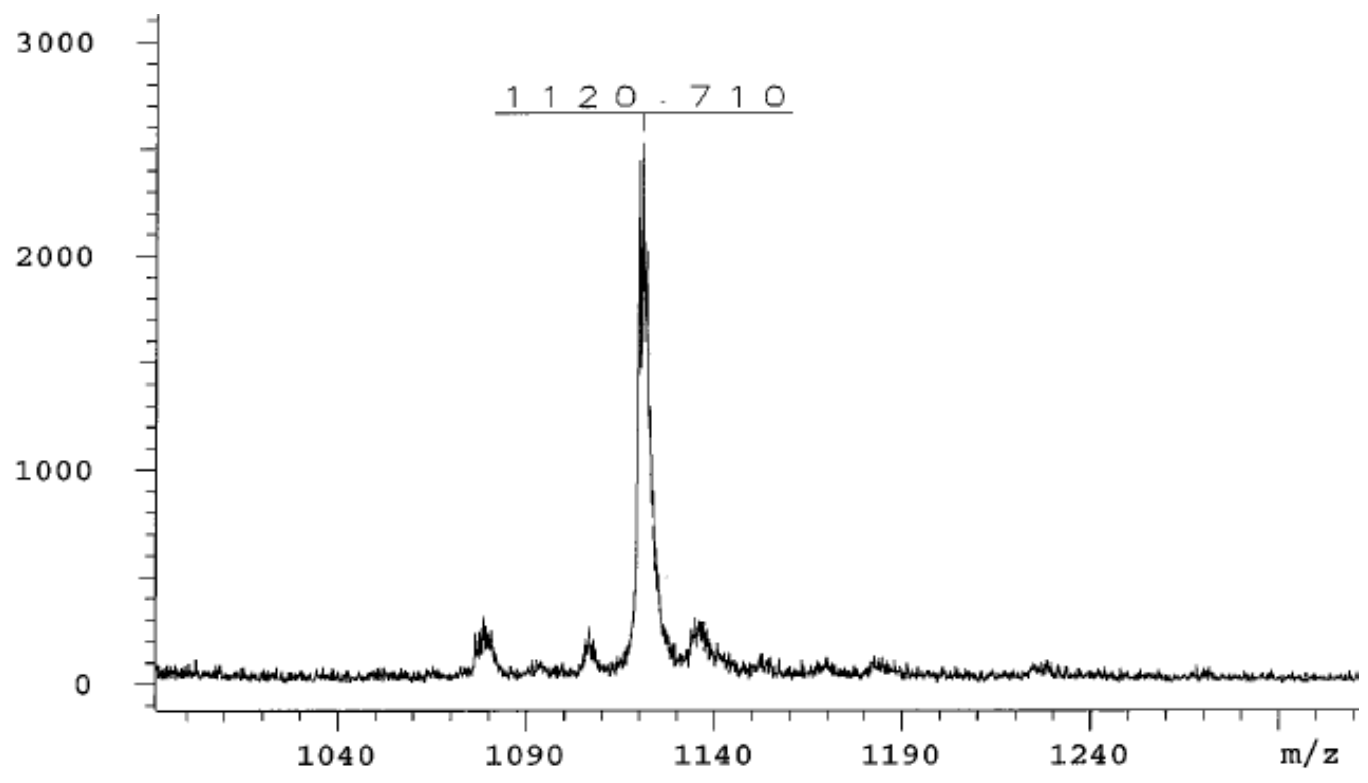

Figure 15 Maldi-tof mass spectrum of bisadduct 12 


\section{Molecular modeling of fulleropyrrolidine bisadducts:}

Models for tethered fulleropyrrolidine bisadducts were built using tools in Spartan'04 by Wavefunction ${ }^{\odot}$ (www.wavefunction.com). The structures were initially optimized using molecular mechanic and then refined with semi-empirical AM1 method.

The computation of AM1 heats of formation of all possible bisadduct isomers having the same addition pattern was conducted to assist in establishing the isomeric structures of fulleropyrrolidine bisadducts. The following only shows the optimized structures with relative lower heats of formation. We used cis and trans to distinguish the stereoisomeric structures.
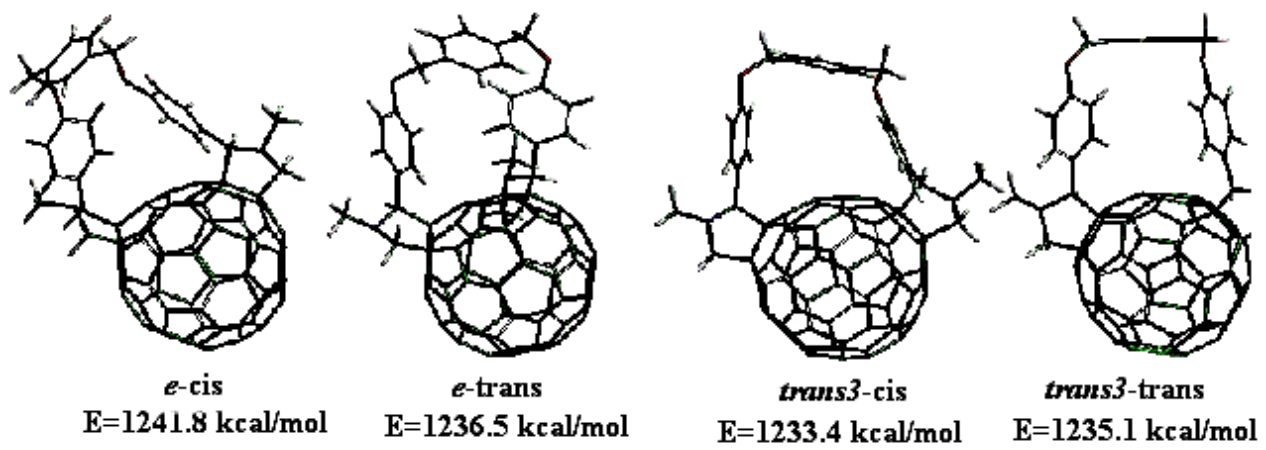

Figure 16 Modeling of bisadducts derived from $p$-xylene tethered bisaldehydes. 

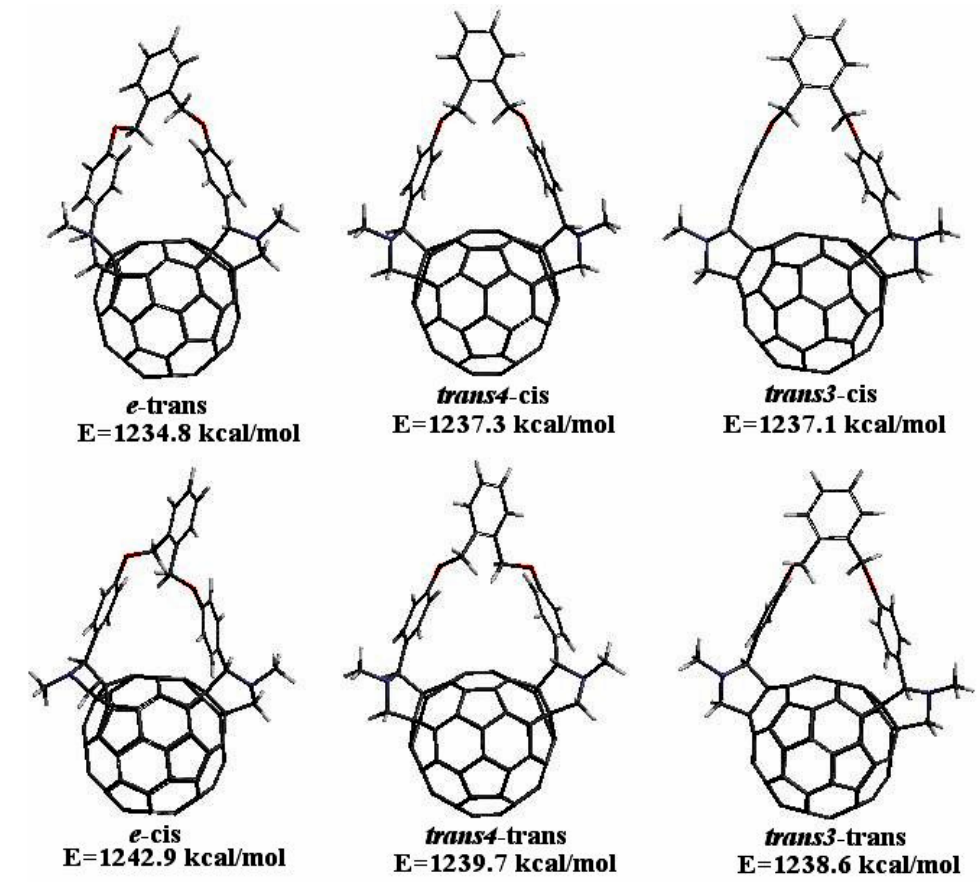

Figure 17 Modeling of bisadducts derived from $o$-xylene tethered bisaldehydes.
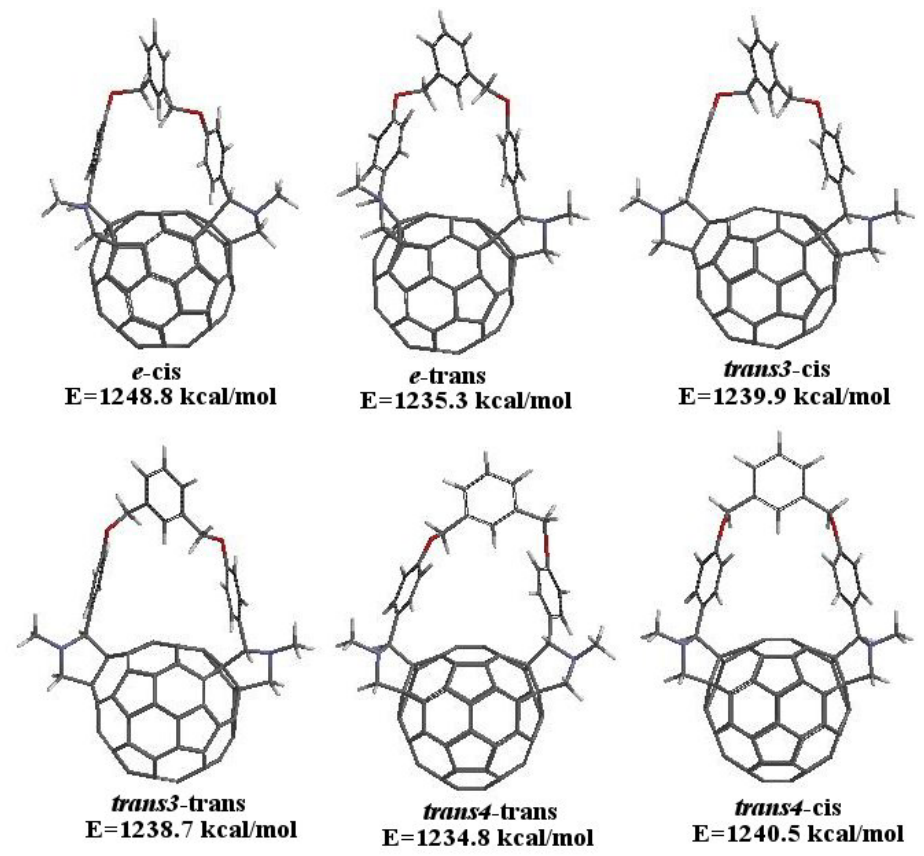

Figure 18 Modeling of bisadducts derived from $m$-xylene tethered bisaldehydes. 

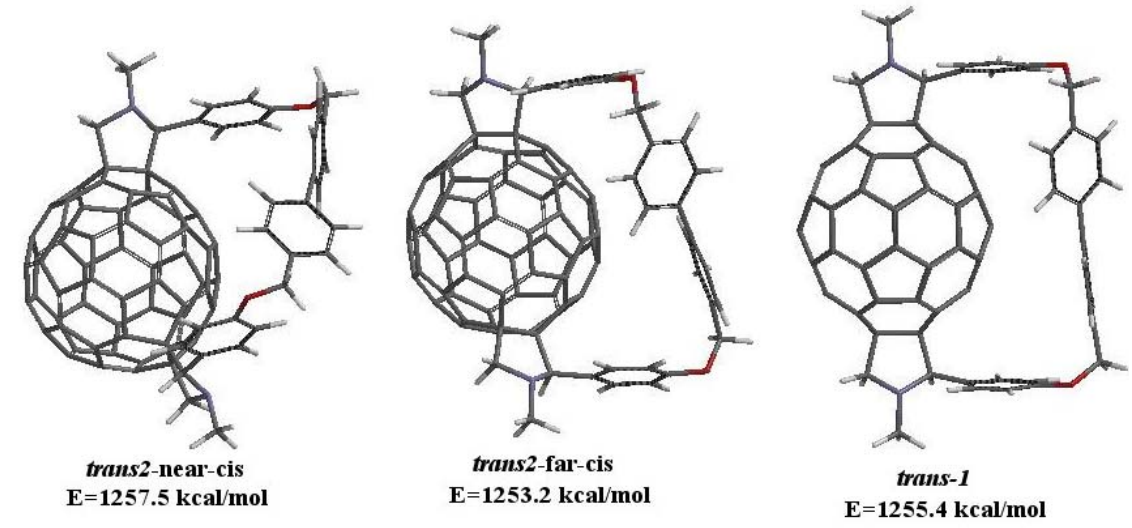

Figure 19 Modeling of fulleropyrrolidine bisadducts derived from biphenyl tethered bisaldehydes 